\title{
Long term follow-up of neoadjuvant chemotherapy for non-small cell lung cancer (NSCLC) investigating early positron emission tomography (PET) scan as a predictor of outcome
}

Perrin E. Romine ${ }^{1}$, Renato G. Martins ${ }^{1,2}$, Keith D. Eaton ${ }^{1,2^{*}}$, Douglas E. Wood ${ }^{1,2}$, Fatemeh Behnia ${ }^{1}$, Bernardo H. L. Goulart ${ }^{1,2}$, Michael S. Mulligan ${ }^{1,2}$, Sarah G. Wallace', Elizabeth Kell ${ }^{2}$, Julie E. Bauman³, Shilpen A. Patel ${ }^{4}$ and Hubert J. Vesselle $e^{1,2}$

\begin{abstract}
Background: Neoadjuvant chemotherapy is effective in improving survival of resectable NSCLC. Based on findings in the adjuvant and metastatic setting, FDG positron emission tomography (PET) scans may offer early prognostic or predictive value after one cycle of induction chemotherapy.

Methods: In this phase II non-randomized trial, patients with AJCC version 6 stage IB to IIIB operable NSCLC were treated with 3 cycles of cisplatin and pemetrexed neoadjuvant chemotherapy. Patients underwent FDG-PET scanning prior to and 18 to 21 days after the first cycle of chemotherapy. Investigators caring for patients were blinded to results, unless the scans showed evidence of disease progression. FDG-PET response was defined prospectively as a $\geq 20 \%$ decrease in the SUV of the primary lesion.

Results: Between October 2005 and February 2010, 25 patients enrolled. Fifty two percent were female, 88\% white, and median age was 62 years. Histology was divided into adenocarcinoma 66\%, not otherwise specified (NOS) 16\%, squamous cell 12\%, and large cell 4\%. Stage distribution was: 16\% IB, 4\% IIB, and 79\% IIIA. Treatment was well tolerated and only one patient had a grade 4 toxicity. The median follow up was 95 months. The 5 year progression free survival (PFS) and overall survival (OS) for the entire population were 54 and $67 \%$, respectively. Eighteen patients had a baseline FDG-PET scan and a repeat scan at day 18-21 available for comparison. Ten patients (56\%) were considered metabolic responders on the day 18-21 FDG-PET scan. Responders had a 5 year PFS and OS of 60 and 70\%, respectively, while the percentage for non-responders was 63 and $75 \%$ ( $p=0.96$ and 0.85 ).
\end{abstract}

Conclusions: This phase II trial did not demonstrate that a PET scan after one cycle of chemotherapy can predict survival outcomes of patients with NSCLC treated with neoadjuvant chemotherapy.

Trial registration: NCT00227539 registered September 28th, 2005.

Keywords: Non-small cell lung cancer, Neoadjuvant chemotherapy, (18F)-FDG PET

\footnotetext{
* Correspondence: kdeaton@u.washington.edu

'University of Washington, School of Medicine, 1959 NE Pacific St, Seattle, WA 98195, USA

${ }^{2}$ Seattle Cancer Care Alliance, 825 Eastlake Ave E, Seattle, WA 98109, USA

Full list of author information is available at the end of the article
}

(c) The Author(s). 2019 Open Access This article is distributed under the terms of the Creative Commons Attribution 4.0 International License (http://creativecommons.org/licenses/by/4.0/), which permits unrestricted use, distribution, and reproduction in any medium, provided you give appropriate credit to the original author(s) and the source, provide a link to the Creative Commons license, and indicate if changes were made. The Creative Commons Public Domain Dedication waiver (http://creativecommons.org/publicdomain/zero/1.0/) applies to the data made available in this article, unless otherwise stated. 


\section{Background}

Despite recent advances in treatment, lung cancer remains the leading cause of cancer-related death in the US and worldwide [1]. Among patients diagnosed with lung cancer, approximately $80 \%$ are diagnosed with non-small cell histology. Management of patients diagnosed with non-metastatic NSCLC includes chemotherapy, radiation therapy, and surgery. Unfortunately, among all patients who undergo surgical resection with curative intent, approximately $40 \%$ will recur [2]. Adjuvant chemotherapy has been demonstrated to improve both progression free and overall survival in resectable NSCLC [3].

Because of this, multiple studies have examined the role of neoadjuvant chemotherapy. Initial studies of neoadjuvant chemotherapy suggested a benefit in patients with mediastinal involvement [4], although long term follow up showed less of a survival benefit than originally reported [5]. Later trials demonstrated a significant 5 year overall survival advantage when using pre-operative chemotherapy in operative NSCLC, including stages IA to IIIB [6-10]. More recent data suggests that there is no difference in 3 year overall survival in patients treated with preoperative vs. postoperative chemotherapy in resectable NSCLC $[3,11,12]$.

Neoadjuvant chemotherapy offers specific advantages over adjuvant treatment, including early treatment of micrometastatic disease and avoidance of treatment delays that may occur due to post-operative complications. Neoadjuvant therapy also offers the opportunity to assess chemotherapy sensitivity and response early during the treatment course and while the primary tumor is still present [13]. Various neoadjuvant regimens have been used in NSCLC, although cisplatin doublet therapy remains standard. Studies have demonstrated superior efficacy of cisplatin and pemetrexed doublet therapy compared with cisplatin and gemcitabine in patients with advanced NSCLC, excluding patients with squamous cell histology. Pemetrexed based regimens are better tolerated than gemcitabine based regimens, motivating our selection of this regimen for patients with adenocarcinoma [14].

Ideally, molecular markers would be used to define early chemotherapy sensitivity and response. However, such markers are not yet available for lung cancer. Alternatively, FDG-PET imaging could be used as a physiological test of response to chemotherapy. FDG-PET is the standard of care in initial staging of NSCLC and in guiding its surgical staging $[15,16]$. Sequential FDG-PET imaging has been evaluated as a metric for response to treatment in NSCLC [17-19]. Studies have indicated that FDG-PET can be used to differentiate radiologic responders from non-responders following one cycle of cisplatin based neoadjuvant chemotherapy [20] and histopathologic response during neoadjuvant erlotinib therapy $[19,21]$ in stage IB-IIIA NSCLC. Chaft et al. published results from a phase II trial of resectable NSCLC patients receiving neoadjuvant chemotherapy with FDG-PET imaging done following two cycles of cisplatin based therapy. The chemotherapy regimen was maintained or changed based on initial metabolic response following two cycles of treatment. This study demonstrated an improved radiographic response by altering treatment based on interval imaging but was not designed to evaluate for survival [22].

Despite increasing research on the use of FDG-PET imaging in NSCLC, studies have failed to consistently demonstrate a predicted survival benefit based on FDG-PET imaging results following neoadjuvant chemotherapy [2330]. Furthermore, these studies fail to address the potential benefit of early quantitative response information as measured by FDG-PET imaging during neoadjuvant treatment. In this phase II study, we aim to test if FDG-PET response early during neoadjuvant chemotherapy predicts for a survival benefit in resectable NSCLC.

\section{Materials and methods Patient population}

This study was conducted with the approval and supervision of the University of Washington Institutional Review Board (IRB) and was registered at clinicaltrials.gov (NCT 00227539.) Eligible patients were defined as having histological or cytological documented NSCLC who were deemed to be candidates for surgical resection with clinical stages IB-IIIB (T4, N0-2) disease. Timing of mediastinal staging with mediastinoscopy (pre-neoadjuvant chemotherapy or at the time of surgery) was decided at the discretion of the surgeon. Patients were required to have an FDG-PET scan with measurable disease as part of initial staging, defined according to RECIST 1.0 criteria [31]. Patients were required to have adequate pulmonary reserve with a predicted FEV1 > $0.8 \mathrm{~L}$ following proposed resection. Patients were also required to have adequate organ function, defined as bilirubin $\leq 1.5 x U L N$, AST and ALT $\leq 3.0 x U L N, C r C l \geq 45 \mathrm{ml} / \mathrm{min}$, platelet count $\geq 100 \times 10^{9} / \mathrm{L}$, and $A N C \geq 1.25 \times 10^{9} / \mathrm{L}$. All patients were age $\geq 18$ with ECOG performance status of 0 or 1. Patients were excluded if they had been treated with any non-FDA approved or investigational drugs within 30 days of enrolling, had unresectable disease or a second primary malignancy, had a malignant pleural effusion, had prior radiation therapy to the chest, had type 1 diabetes (type 2 diabetes patients were included if glucose levels were well controlled), were pregnant or nursing, were unable to interrupt aspirin or NSAID therapy for 5 days, or if they were unable to take any of the required study drugs, including corticosteroids, folic acid, or vitamin B12. All patients provided informed consent. In total, 25 patients were recruited to this phase II study.

\section{Preoperative treatment}

All patients were treated with a maximum of three cycles of neoadjuvant cisplatin $75 \mathrm{mg} / \mathrm{m}^{2}$ and pemetrexed 
$500 \mathrm{mg} / \mathrm{m}^{2}$ on day 1 of a 21 day cycle. Following the phase III trial by Scagliotti et al. demonstrating inferior survival for squamous cell patients treated with cisplatin/ pemetrexed, patients with squamous cell pathology were excluded [14]. Interval FDG-PET scans were not used to make treatment decisions, except when scans showed disease progression precluding surgical cure, in which case patients were withdrawn from the study.

\section{Radiologic analysis}

The initial FDG-PET scan was done within 4 weeks preceding the first cycle of neoadjuvant chemotherapy with concomitant CT scan of the chest and upper abdomen. Patients were considered eligible regardless of the performing location of their initial FDG-PET and CT scan. Repeat FDG-PET scans were performed at the Seattle Cancer Care Alliance/ University of Washington Medical Center after the first (between day 18 and 21) and third cycle (day 64) of neoadjuvant chemotherapy. Repeat CT of the chest and abdomen was performed after the third cycle of chemotherapy prior to surgery. All PET imaging done at SCCA/UWMC was performed on a GE Discovery DSTE PET/CT system (GE Medical Systems, Waukesha, WI).

Prior to FDG-PET imaging, patients fasted for $12 \mathrm{~h}$. Immediately prior to imaging, patients were confirmed to have blood glucose measurements between 80 and 150 $\mathrm{mg} / \mathrm{dl}$. Patients received an IV injection of $10 \mathrm{mCi} \mathrm{F}-18$ FDG followed by a 60-min period of rest. An initial emission scan was done over 5 fields-of-view of 7 min duration each, encompassing the head, neck, thorax, abdomen, and pelvis. A $2.5 \mathrm{~mm}$ non-contrast axial CT scan was performed for localization and attenuation correction. Target lesions on CT imaging were selected on the basis of size (longest diameter) and their suitability for accurate repeated measurements.

\section{Quantitative analysis}

FDG-PET standardized uptake value (SUV) was evaluated in the primary tumor of each subject. A SUV was defined as the time averaged tissue activity $\mathrm{C}(\mu \mathrm{Ci} / \mathrm{ml})$, from 60 to $67 \mathrm{~min}$ following injection, divided by the injected dose ID $(\mathrm{mCi})$ per kilogram of patient body weight. The maximum SUV value (SUVmax) was used for the primary tumor response, defined as the maximum pixel value within a region of interest (ROI) encompassing the entire primary lesion on the SUV images. Percentage SUV changes between initial and subsequent FDG-PET scans were calculated based on primary tumor SUVmax.

\section{Statistical analysis}

The primary objective of this trial was to evaluate the potential effectiveness of FDG-PET after one cycle of chemotherapy in predicting radiographic response to neo-adjuvant chemotherapy in patients with operable stage IB to IIIB
NSCLC. Previous work in a patient population with advanced disease indicated that a decrease in tumor SUV of $20 \%$ or greater was associated with radiographic response with a PPV of 0.71 and a NPV of 0.96 [27]. A sample size of 35 was chosen to provide sufficient power to evaluate different FDG-PET response thresholds under varying assumptions of response rate by CT. A lower than predicted CT response rate observed in this study precluded analysis of different thresholds and we therefore analyzed the data based on the $20 \%$ threshold. Secondary outcomes were PFS and OS.

All patients were followed at 3-month intervals for the initial 2 years, then at 6-month intervals for a subsequent 3 years. A $20 \%$ decrease in SUV of the primary tumor as measured by FDG-PET was utilized as the threshold for metabolic response, given prior work by Weber et al. demonstrating that this was associated with radiographic response [27]. Radiographic response was defined per RECIST 1.0 criteria [31]. Complete response was defined as complete resolution of all radiological abnormalities. Partial response was defined as at least a $30 \%$ decrease in the sum of the longest diameter of the target lesions. Progressive disease was defined as at least a $20 \%$ increase in the sum of the longest diameter of the target lesion. Stable disease was defined in patients that did not qualify as partial response or progressive disease. $P$ values less than 0.05 were considered significant. PFS and OS were assessed using the Kaplan-Meier log-rank method. Post-hoc analysis was performed further evaluating survival trends based on metabolic response on post-chemotherapy FDG-PET, excluding patients with squamous cell histology, and utilizing a 30\% decrease in SUV as the threshold for metabolic response per PERCIST criteria [32].

\section{Results}

\section{Patient characteristics}

Twenty-five patients were enrolled between October 2005 and February 2010. Due to slow recruitment, this study was closed prior to enrolling the goal sample size. Baseline patient characteristics are summarized in Table 1. One patient was excluded from analysis following surgical resection due to reclassification of her tumor histology as carcinoid (despite two independent reviews indicating NSCLC in the initial biopsy). Fifty percent of patients underwent mediastinal staging prior to neoadjuvant chemotherapy; the remainder of patients underwent mediastinal staging following neoadjuvant chemotherapy. Of the 19 patients with Stage IIIA disease, 11 (58\%) had confirmed pathologic N2 disease prior to neoadjuvant treatment. Of the remaining eight patients, three had confirmed N2 disease following neoadjuvant treatment while five had confirmed N0-N1 disease. Treatment was well tolerated. One patient had a grade 4 toxicity involving 
Table 1 Patient Characteristics

\begin{tabular}{ll}
\hline Characteristic & Number \\
\hline Female sex, n (\%) & $13(52)$ \\
Median age, years & 62 \\
Histologic Subtype, n (\%) & $16(67)$ \\
Adenocarcinoma & $3(13)$ \\
Squamous cell carcinoma & $4(16)$ \\
NSCLC, not otherwise specified & $1(4)$ \\
Large cell carcinoma & \\
Clinical stage, n (\%) & $4(16)$ \\
IB & 0 \\
IIA & $1(4)$ \\
IIB & $19(79)$ \\
IIIA & \\
Smoking, n (\%) & $23(92)$ \\
Yes & $2(8)$ \\
No & \\
PS, n (\%) & $19(76)$ \\
0 & $4(16)$ \\
\hline
\end{tabular}

NSCLC non-small cell lung cancer, PS performance status

a cerebral vascular accident (CVA); this patient was subsequently withdrawn from the study. Nine patients had grade three toxicities, of which six were felt to be directly related to the study treatment (Table 2). Three patients withdrew from the study: one due to the previously mentioned CVA, two due to disease progression while on neoadjuvant treatment. In total, 18 patients were eligible for final analysis, of which 14 underwent definitive surgical resection (Fig. 1). Four patients did not undergo surgical resection due to disease progression following neoadjuvant chemotherapy.

Table 2 Adverse Events

\begin{tabular}{lll}
\hline Toxicity Category & Grade & Number (\%) \\
\hline Fatigue & 3 & $7(28)$ \\
Pain & 3 & $2(8)$ \\
Metabolic/Laboratory & 3 & $1(4)$ \\
Pulmonary & 3 & $3(12)$ \\
Gastrointestinal & 3 & $4(16)$ \\
Dermatologic/skin & 3 & $2(8)$ \\
Neurologic & 4 & $1(4)$ \\
Allergy/lmmunologic & 3 & $1(4)$ \\
\hline No: Maximum grade per paten per
\end{tabular}

Note: Maximum grade per patient per body system presented. Number of evaluable patients: 25 . Total number of patients with grade 3/4 toxicities: 10 (patients with multiple toxicities and are listed separately above)
Radiologic and pathologic response

Of the eighteen evaluable patients, 14 (78\%) had stable disease on repeat chest CT following 3 cycles of neoadjuvant therapy, 2 (11\%) had a partial response, and one (6\%) had progressive disease. One patient did not have baseline chest CT images available for comparison. None of the fourteen patients who underwent definitive surgical resection had a pathologic complete response following 3 cycles of neoadjuvant chemotherapy.

\section{Metabolic response}

Ten patients $(56 \%)$ were considered metabolic responders on day 18-21 FDG-PET, using a $20 \%$ reduction in SUV as a threshold. Nine patients (50\%) were considered metabolic responders on day 18-21 FDG-PET when the SUV threshold was adjusted to $30 \%$. There was a median $28.9 \%$ decrease in interval SUV, ranging from an $80.5 \%$ decrease to a $46.3 \%$ increase. In total, 17 patients had complete FDG-PET and CT imaging. Of the 10 patients considered early metabolic responders, 8 had stable disease on repeat chest CT following 3 cycles of neoadjuvant therapy while 2 had a partial response. Of the 7 patients considered metabolic non-responders, 6 had stable disease on repeat CT imaging while 1 had progressive disease. Among the 4 patients with disease progression following neoadjuvant chemotherapy, 2 were deemed early metabolic responders.

\section{Progression free survival and overall survival}

The median followup of study participants was 95 months. The 5 year PFS and OS for the entire population were 54 and $67 \%$, respectively. The overall survival curve for the entire cohort is shown in Fig. 2. When stratified by metabolic response, PFS at 5 years for responders and non-responders was 60 and 63\%, respectively. OS at 5 years for responders was $70 \%$, while the percentage for non-responders was $75 \%$. Kaplan-Meier curves for PFS and OS are shown in Figs. 3 and 4 ( $p$-values 0.96 and 0.85 , respectively). In an intention to treat analysis, PFS and OS of non-responders decreased to 50 and $64 \%$ at 5 years, respectively, with no change in Kaplan-Meier log-rank statistics (p-value 0.62 and 0.61). PFS and OS trends were re-analyzed in a post-hoc analysis excluding patients with squamous cell histology ( $\mathrm{p}$-value 0.91 and 0.89 , respectively) and using a $30 \%$ decrease in SUV as the threshold for metabolic response (p-value 0.65 and 0.33 , respectively). PFS and OS trends were further re-analyzed utilizing metabolic response following completion of neoadjuvant chemotherapy (also utilizing a 20\% reduction in SUV as a threshold for metabolic response). There was no significant difference in either survival trend ( $p$-value 0.55 and 0.50 , respectively).

\section{Discussion}

FDG-PET imaging has the potential to provide early quantitative response information during neoadjuvant treatment of 


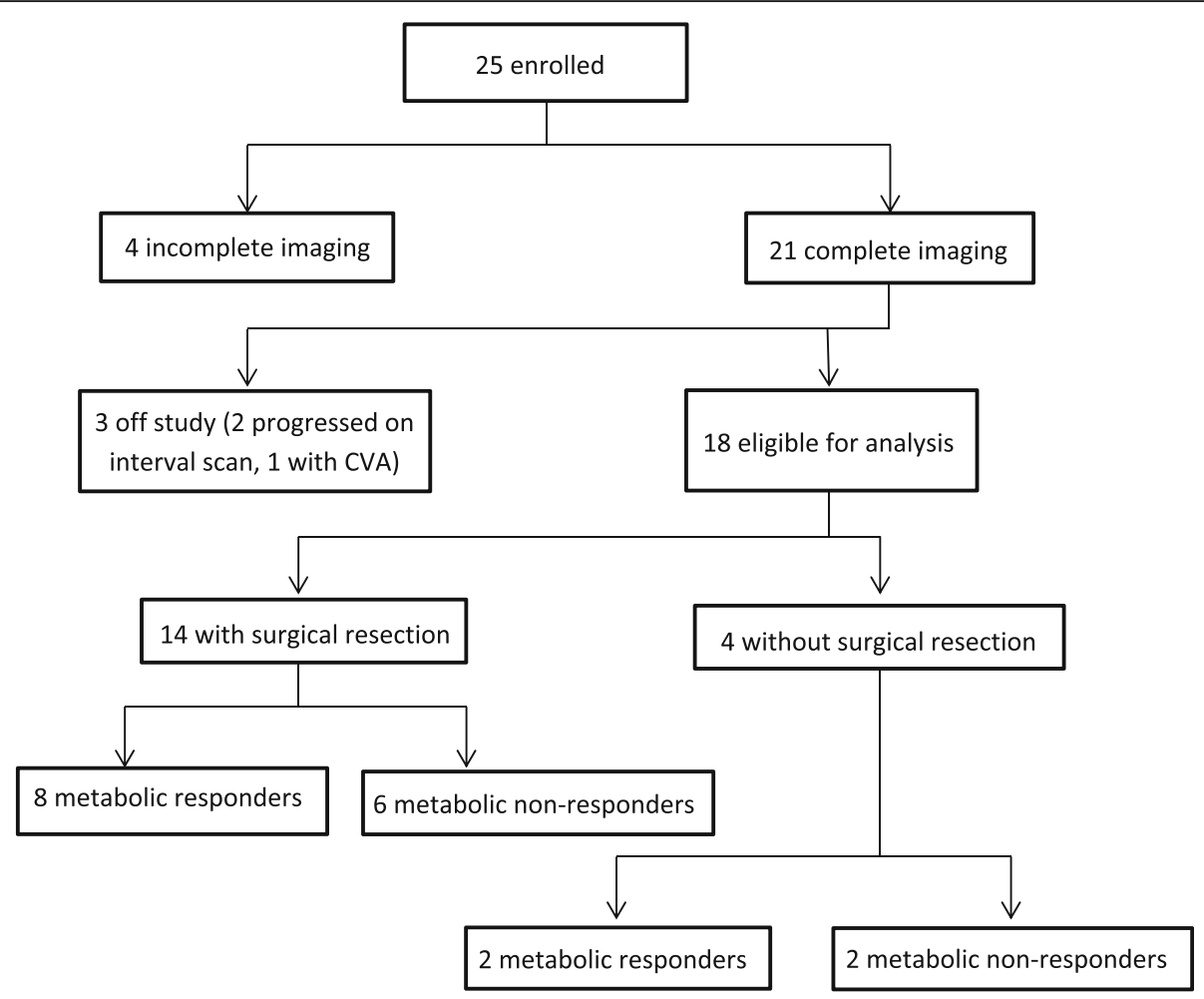

Fig. 1 CONSORT flow diagram; CVA, cerebral vascular accident

NSCLC. Our aim was to evaluate imaging done following one cycle of neoadjuvant treatment as a means of measuring early prognostic information in NSCLC. Studies have shown that FDG-PET imaging during neoadjuvant treatment (after one or two cycles) is predictive of later radiographic response, and can possibly be used to adapt chemotherapy regimen [20, 22]. Hoekstra et al. demonstrate that metabolic response on FDG-PET imaging done after one cycle of platinum-based neoadjuvant chemotherapy is predictive of prognosis [26]. Contrary to this, our study did not demonstrate a clear association between early PET assessment with later $\mathrm{CT}$ response. No change in survival was observed when patients with squamous cell histology were excluded from the analysis or when the threshold for metabolic response was adjusted to $30 \%$.

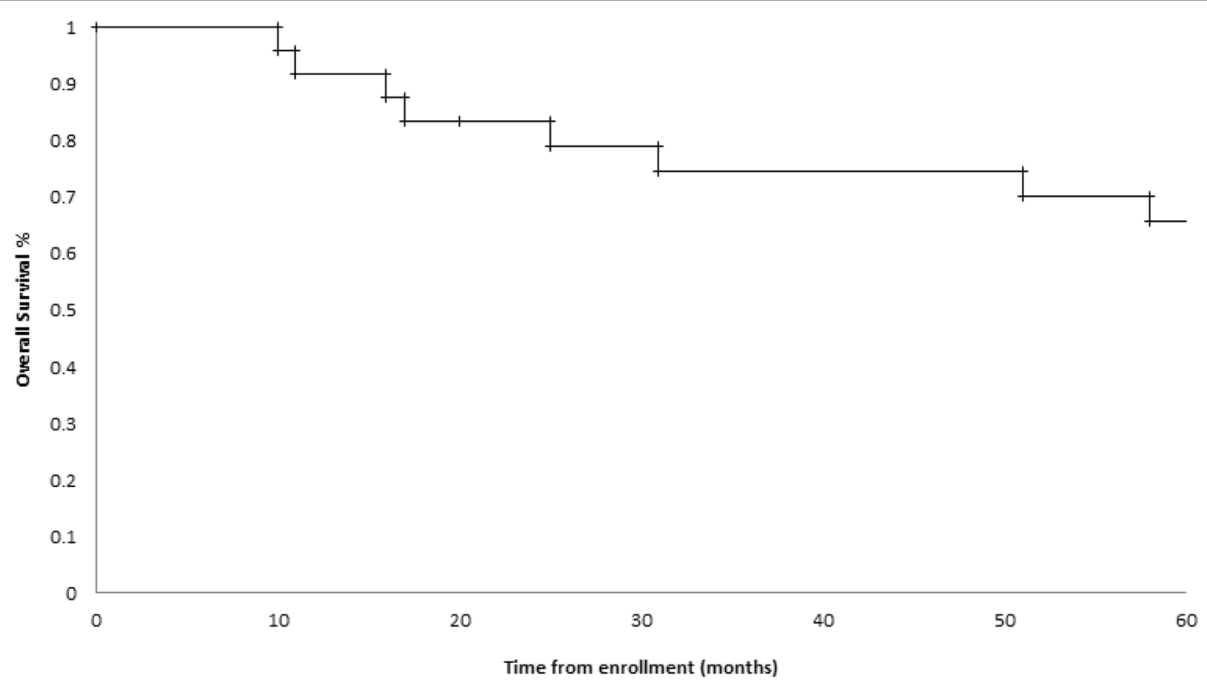

Fig. 2 Overall Survival of the entire cohort censored at 5 years 


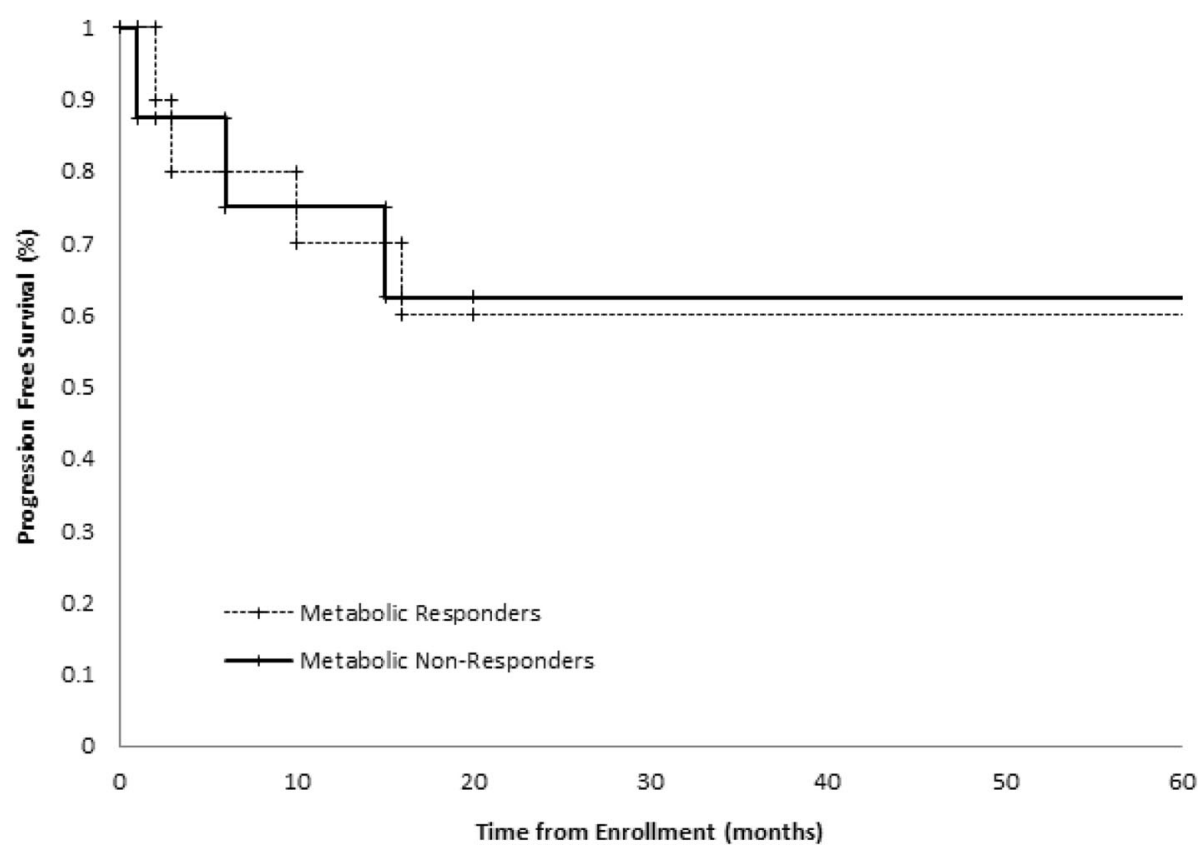

Fig. 3 Kaplan-Meier progression free survival curve, stratified by metabolic response on interval FDGPET imaging. Response was defined as $\geq 20 \%$ decrease in SUV of the dominant lesion

There are multiple factors that may have influenced our results. This study is underpowered to show potential clinically significant survival differences. However, the survival curves shown in Figs. 3 and 4 overlap, suggesting that a significant survival difference is unlikely. Five-year overall survival of the accrued cohort was $67 \%$.
This remains higher than expected based on reported large scale meta-analyses of NSCLC, even when accounting for the predominance of patients with Stage IIIA disease in our cohort and the expected survival benefit from neoadjuvant chemotherapy [7, 33]. While this likely arose by chance and reflects the small size of

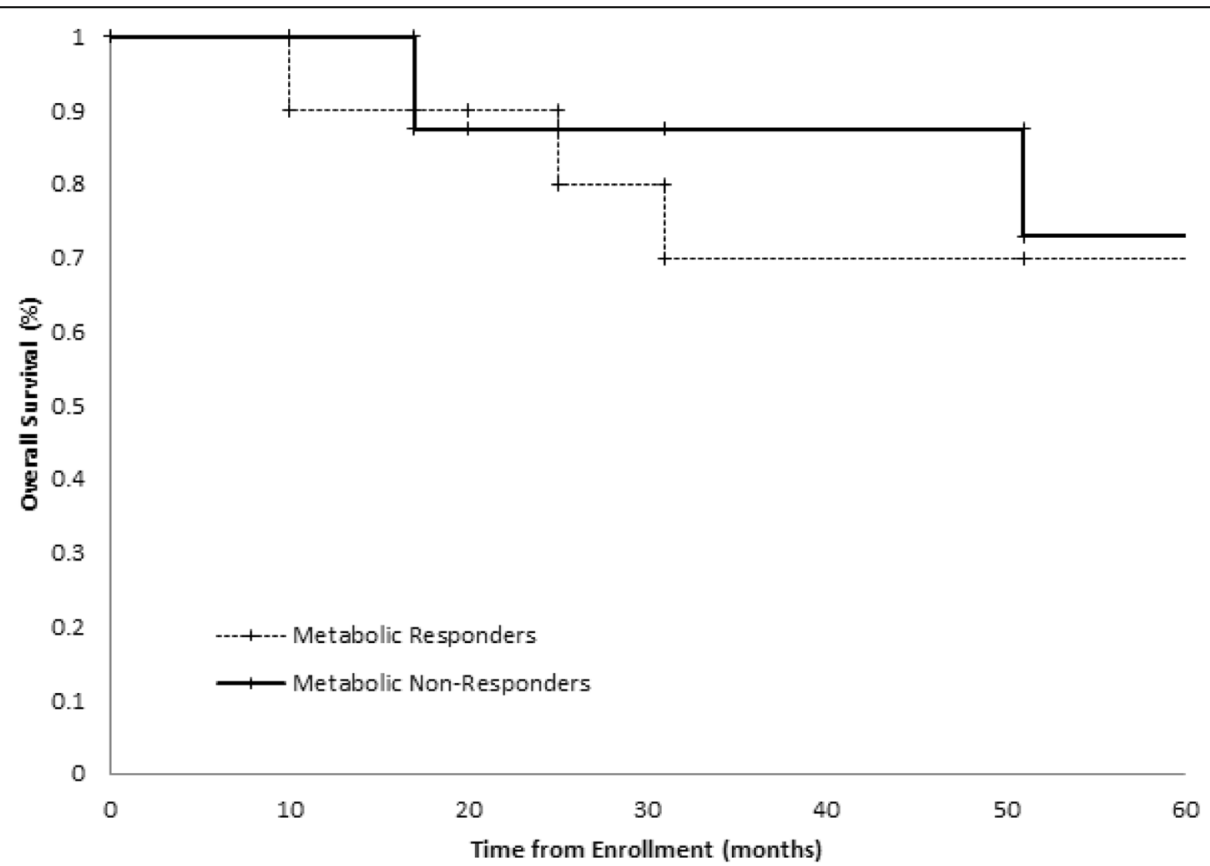

Fig. 4 Kaplan-Meier overall survival curve, stratified by radiologic response on interval FDG-PET imaging. Response was defined as $\geq 20 \%$ decrease in SUV of the dominant lesion 
our cohort and the single institutional nature of this trial, this disparity may impact the predictive nature of early FDG-PET imaging within this study cohort.

In addition, this trial included patients with initial FDG-PET imaging done at other institutions. Test-retest repeatability studies of multicenter FDG-PET scans have demonstrated that a decrease in SUVmax by $30 \%$ in scans done at the same institution is unlikely to reflect variability in measurement [34]. However, our study design, while representative of clinical practice, could lead to further variability in SUV quantifications.

As this study illustrates, the utility of early FDG-PET scanning during neo-adjuvant therapy, optimal timing, and the criteria for imaging response are areas that require further research. By repeating imaging following only one cycle of neoadjuvant chemotherapy, we hoped to demonstrate early predictive information that could be used to drive treatment choices and reduce unnecessary treatment related morbidity. Prior work following patients with inoperable NSCLC with weekly FDG-PET scans during the first 7 weeks of chemotherapy has demonstrated that prognostic information can be determined as early as 3 weeks [35]. However, it remains unclear if these results can be extrapolated to patients with operable NSCLC receiving neoadjuvant therapy.

Numerous methods are currently used to assess tumor response as measured by FDG-PET imaging. By defining metabolic response prospectively, rather than post-hoc, we hoped to avoid bias in interpreting FDG-PET response. SUV is widely used in the clinical setting across practice settings and does not require dynamic data acquisition or complex data analysis. A response based on SUV change, therefore, offers significant advantages when considering clinical implementation. A $20 \%$ threshold was chosen based on prior work demonstrating $10 \%$ reproducibility in SUV measurements; by using $20 \%$ we aimed to capture patients with a change in SUV that was greater that two times the standard deviation [27]. However, it is possible that a more stringent definition of metabolic response, such as the dynamic metabolic rate of glucose used by Hoekstra et al., is necessary to accurately predict prognostic information [26].

Multiple studies have looked at the predictive value of FDG-PET following, rather than during, neoadjuvant treatment of NSCLC. Results from these studies have been variable, with some studies demonstrating an association between metabolic response and survival, while others fail to demonstrate a predicted survival benefit [23-25, 28-30]. While the results are disparate, it should be noted that study design, choice of neoadjuvant therapy, cohort size, and clinical stage of included patients varied greatly between these studies. In particular, FDG-PET metabolic response definitions are highly inconsistent, ranging from a significant decrease in the metabolism of the index lesion by direct comparison as determined by a reading nuclear radiologist [29] to absolute SUV cutoffs on follow up imaging $[23,25]$ to variable rates of SUV change on follow up imaging $[24,28,30]$. These inconsistencies may in part explain the variable results seen across these studies. Our study, while designed to evaluate early FDG-PET response, did not demonstrate a predicted survival benefit when analyzing FDG-PET imaging done following completion of neoadjuvant therapy.

In conclusion, this study failed to demonstrate that early FDG-PET imaging after one cycle of neoadjuvant chemotherapy can predict survival outcomes of patients with NSCLC. As utilization of neoadjuvant treatment changes in NSCLC patients, further work is needed to better understand the optimal timing and method to assess early chemotherapy sensitivity and response.

\section{Abbreviations}

AJCC: American Joint Committee on Cancer; ALT: Alanine aminotransferase: ANC: Absolute neutrophil count; AST: Aspartate aminotransferase; CrCL: Creatinine clearance; CT: Computed tomography; CVA: Cerebral vascular accident; ECOG: Eastern cooperative oncology group; FDA: Food and drug administration; FDG: Fluorodeoxyglucose; FEV: Forced expiratory volume; ID: Injected dose; IRB: Institutional review board; NOS: Not otherwise specified; NSAID: Non-steroidal anti-inflammatory drug; NSCLC: Non-small cell lung cancer; OS: Overall survival; PERCIST: Positron emission tomography response criteria in solid tumors; PET: Positron emission tomography; PFS: Progression free survival; RECIST: Response evaluation criteria in solid tumors; ROI: Region of interest; SUV: Standardized uptake value; ULN: Upper limit of normal

\section{Acknowledgements}

The authors would like to thank the physicians, technologists, and physicists from the University of Washington Medical Center/Seattle Cancer Care Alliance who helped make this study possible. We also thank the patients that volunteered for this study.

\section{Funding}

This study was funded in part by patient donations made to Thoracic Head and Neck Oncology Research Program of the Seattle Cancer Care Alliance. This work was supported by Eli Lilly protocol number 6228, grant number H3E-US-X043. Eli Lilly was not involved in the design of this study, in the collection, analysis, interpretation of data, or in the writing of this manuscript.

Availability of data and materials

The datasets used and/or analyzed during the current study are available from the corresponding author on reasonable request.

\section{Authors' contributions}

$R M, K E, D W, F B, B G, M M, S W, E K, J B, S P$, and HV participated in study design, patient recruitment, and data collection. PR participated in data collection and analysis and was a major contributor in writing the manuscript. KE, BG, and SW further contributed to data analysis. All authors read and approved the final manuscript.

\section{Ethics approval and consent to participate}

This study was approved under the University of Washington/Seattle Cancer Care Alliance Ethics board. All patients gave written informed consent prior to enrolling in this trial.

Consent for publication

Not applicable, no individual data/images presented.

\section{Competing interests}

We wish to draw the attention of the Editor to the following facts which may be considered as potential conflict of interest:

1. At the time of study recruitment Dr. Renato Martins was part of the Eli Lilly Speaker's Bureau and has received honorarium from Eli Lilly. He 
has not received honorarium for greater than 2 years at this time and is no longer part of the Speaker's Bureau.

2. Dr. Hubert Vesselle is a paid consultant for MIM software, Inc. This software was not used as a tool in the NeoPET trial.

The remainder of the authors confirm that they have no known conflicts of interest associated with this publication and there has been no significant financial support aside from what is listed above and in the acknowledgements section that could have influenced its outcome.

\section{Publisher's Note}

Springer Nature remains neutral with regard to jurisdictional claims in published maps and institutional affiliations.

\begin{abstract}
Author details
'University of Washington, School of Medicine, 1959 NE Pacific St, Seattle, WA 98195, USA. ${ }^{2}$ Seattle Cancer Care Alliance, 825 Eastlake Ave E, Seattle, WA 98109, USA. ${ }^{3}$ University of Arizona, Tucson, AZ, USA. ${ }^{4}$ Grail Inc., Menlo Park, CA, USA.
\end{abstract}

Received: 13 July 2018 Accepted: 7 January 2019

Published online: 14 January 2019

\section{References}

1. Siegel RL, Miller KD, Jemal A. Cancer statistics, 2017. CA Cancer J Clin. 2017;67:7-30

2. Sugimura H, Nichols FC, Yang P, Allen MS, Cassivi SD, Deschamps C, et al. Survival after recurrent non small-cell lung cancer after complete pulmonary resection. Ann Thorac Surg. 2007;83:409-17.

3. Lim E, Harris G, Patel A, Adachi I, Edmonds L, Song F. Preoperative versus postoperative chemotherapy in patients with resectable non-small cell lung cancer: systematic review and indirect comparison meta-analysis of randomized trials. J Thorac Oncol. 2009;4:1380-8.

4. Rosell R, Gómez-Codina J, Camps C, Maestre J, Padille J, Cantó A, et al. A randomized trial comparing preoperative chemotherapy plus surgery with surgery alone in patients with non-small-cell lung cancer. N Engl J Med. 1994;330:153-8.

5. Rosell R, Gómez-Codina J, Camps C, Javier Sánchez J, Maestre J, Padilla J, et al. Preresectional chemotherapy in stage IIIA non-small-cell lung cancer: a 7-year assessment of a randomized controlled trial. Lung Cancer. 1999;26:7-14.

6. Berghmans T, Paesmans M, Meert AP, Mascaux C, Lothaire P, Lafitte JJ, et al. Survival improvement in resectable non-small cell lung cancer with (neo)adjuvant chemotherapy: results of a meta-analysis of the literature. Lung Cancer. 2005;49:13-23.

7. Burdett SS, Stewart LA, Rydzewska L. Chemotherapy and surgery versus surgery alone in non-small cell lung cancer. Cochrane Database Syst Rev. 2007;18:CD006157.

8. Song W-A, Zhou N-K, Wang W, Chu X-Y, Liang C-Y, Tian X-D, et al. Survival benefit of neoadjuvant chemotherapy in non-small cell lung cancer: an updated meta-analysis of 13 randomized control trials. J Thorac Oncol. 2010;5:510-6.

9. NSCLC Meta-analysis Collaborative Group. Preoperative chemotherapy for non-small-cell lung cancer: a systematic review and meta-analysis of individual participant data. Lancet. 2014;383:1561-71.

10. Mouillet G, Monnet E, Milleron B, Puyraveau M, Quoix E, David P, et al. Pathologic complete response to preoperative chemotherapy predicts cure in early-stage non-small-cell lung cancer: combined analysis of two IFCT randomized trials. J Thorac Oncol. 2012;7:841-9.

11. Westeel V, Quoix E, Puyraveau M, Lavolé A, Braun D, Laporte S, et al. A randomised trial comparing preoperative to perioperative chemotherapy in early-stage non-small-cell lung cancer (IFCT 0002 trial). Eur J Cancer. 2013; 49:2654-64.

12. Howington JA, Blum MG, Chang AC, Balekian AA, Murthy SC. Treatment of stage I and II non-small cell lung cancer: diagnosis and management of lung cancer, 3rd ed: American College of Chest Physicians evidence-based clinical practice guidelines. Chest. 2013;143(5 Suppl):e278S-313S.

13. Baik CS, Vallières E, Martins RG. The role of chemotherapy in the management of stage IIIA non-small cell lung cancer. Am Soc Clin Oncol Educ Book. 2013;2013:320-5.

14. Scagliotti GV, Parikh P, von Pawel J, Biesma B, Vansteenkiste J, Manegold C, et al. Phase III study comparing cisplatin plus gemcitabine with cisplatin plus pemetrexed in chemotherapy-naive patients with advanced-stage nonsmall-cell lung cancer. J Clin Oncol. 2008;26:3543-51.

15. Vesselle H, Pugsley JM, Vallières E, Wood DE. The impact of fluorodeoxyglucose F 18 positron-emission tomography on the surgical staging of non-small cell lung cancer. J Thorac Cardiovasc Surg. 2002;124:511-9.

16. Gould MK, Kuschner WG, Rydzak CE, Maclean CC, Demas AN, Shigemitsu H, et al. Test performance of positron emission tomography and computed tomography for mediastinal staging in patients with non-small-cell lung cancer: a meta-analysis. Ann Intern Med. 2003;139:879-92.

17. Lee DH, Kim S-K, Lee H-Y, Lee SY, Park SH, Kim HY, et al. Early prediction of response to first-line therapy using integrated 18F-FDG PET/CT for patients with advanced/ metastatic non-small cell lung cancer. J Thorac Oncol. 2009;4:816-21.

18. Usmanij EA, de Geus-Oei L-F, Troost EGC, Peters-Bax L, van der Heijden EHFM, Kaanders JHAM, et al. 18F-FDG PET early response evaluation of locally advanced non-small cell lung cancer treated with concomitant chemoradiotherapy. J Nucl Med. 2013;54:1528-34.

19. Aukema TS, Kappers I, Olmos RAV, Codrington HE, van Tinteren $H$, van Pel $R$, et al. Is 18F-FDG PET/CT useful for the early prediction of histopathologic response to neoadjuvant erlotinib in patients with non-small cell lung cancer? J Nucl Med. 2010;51:1344-8.

20. Crandall JP, Tahari AK, Juergens RA, Brahmer JR, Rudin CM, Esposito G, et al. A comparison of FLT to FDG PET/CT in the early assessment of chemotherapy response in stages IB-IIIA resectable NSCLC. EJNMMI Res. 2017;7:8.

21. van Gool MH, Aukema TS, Schaake EE, Rijna H, Valdés Olmos RA, van Pel R, et al. Timing of metabolic response monitoring during erlotinib treatment in non-small cell lung cancer. J Nucl Med. 2014;55:1081-6.

22. Chaft JE, Dunphy M, Naidoo J, Travis WD, Hellmann M, Woo K, et al. Adaptive neoadjuvant chemotherapy guided by (18)F-FDG PET in Resectable non-small cell lung cancers: the NEOSCAN trial. J Thorac Oncol. 2016:11:537-44.

23. Decoster $L$, Schallier D, Everaert $H$, Nieboer K, Meysman M, Neyns B, et al. Complete metabolic tumour response, assessed by 18-fluorodeoxyglucose positron emission tomography (18FDG-PET), after induction chemotherapy predicts a favourable outcome in patients with locally advanced non-small cell lung cancer (NSCLC). Lung Cancer. 2008;62:55-61.

24. Eschmann SM, Friedel G, Paulsen F, Reimold M, Hehr T, Budach W, et al. Repeat 18F-FDG PET for monitoring neoadjuvant chemotherapy in patients with stage III non-small cell lung cancer. Lung Cancer. 2007;55:165-71.

25. Hellwig D, Graeter TP, Ukena D, Georg T, Kirsch C-M, Schäfers H-J. Value of F-18-fluorodeoxyglucose positron emission tomography after induction therapy of locally advanced bronchogenic carcinoma. J Thorac Cardiovasc Surg. 2004;128:892-9.

26. Hoekstra CJ, Stroobants SG, Smit EF, Vansteenkiste J, van Tinteren H, Postmus PE, et al. Prognostic relevance of response evaluation using [18F]2-fluoro-2-deoxy-D-glucose positron emission tomography in patients with locally advanced non-small-cell lung cancer. J Clin Oncol. 2005;23:8362-70.

27. Weber WA, Petersen V, Schmidt B, Tyndale-Hines L, Link T, Peschel C, et al. Positron emission tomography in non-small-cell lung cancer: prediction of response to chemotherapy by quantitative assessment of glucose use. J Clin Oncol. 2003;21:2651-7.

28. Roy S, Pathy S, Kumar R, Mohanti BK, Raina V, Jaiswal A, et al. Efficacy of 18F-fluorodeoxyglucose positron emission tomography/computed tomography as a predictor of response in locally advanced non-small-cell carcinoma of the lung. Nucl Med Commun. 2016;37:129-38.

29. Tanvetyanon T, Eikman EA, Sommers E, Robinson L, Boulware D, Bepler G. Computed tomography response, but not positron emission tomography scan response, predicts survival after neoadjuvant chemotherapy for resectable non-small-cell lung cancer. J Clin Oncol. 2008;26:4610-6.

30. Pöttgen $C$, Levegrün $S$, Theegarten $D$, Marnitz $S$, Grehl S, Pink R, et al. Value of 18F-fluoro-2-deoxy-D-glucose-positron emission tomography/computed tomography in non-small-cell lung cancer for prediction of pathologic response and times to relapse after neoadjuvant chemoradiotherapy. Clin Cancer Res. 2006;12:97-106.

31. Therasse P, Arbuck SG, Eisenhauer EA, Wanders J, Kaplan RS, Rubinstein L, et al. New guidelines to evaluate the response to treatment in solid tumors. J Natl Cancer Inst. 2000;92:205-16.

32. Wahl RL, Jacene $H$, Kasamon $Y$, Lodge MA. From RECIST to PERCIST: evolving considerations for PET response criteria in solid tumors. J Nucl Med. 2009;50(Suppl 1):122S-50S.

33. Goldstraw P, Chansky K, Crowley J, Rami-Porta R, Asamura H, Eberhardt WEE, et al. The IASLC lung Cancer staging project: proposals for revision of the 
TNM stage groupings in the forthcoming (eighth) edition of the TNM classification for lung Cancer. J Thorac Oncol. 2016;11:39-51.

34. Weber WA, Gatsonis CA, Mozley PD, Hanna LG, Shields AF, Aberle DR, et al. Repeatability of 18F-FDG PET/CT in advanced non-small cell lung Cancer: prospective assessment in 2 multicenter trials. J Nucl Med. 2015;56:1137-43.

35. Nahmias C, Hanna WT, Wahl LM, Long MJ, Hubner KF, Townsend DW. Time course of early response to chemotherapy in non-small cell lung cancer patients with 18F-FDG PET/CT. J Nucl Med. 2007;48:744-51.

Ready to submit your research? Choose BMC and benefit from:

- fast, convenient online submission

- thorough peer review by experienced researchers in your field

- rapid publication on acceptance

- support for research data, including large and complex data types

- gold Open Access which fosters wider collaboration and increased citations

- maximum visibility for your research: over $100 \mathrm{M}$ website views per year

At BMC, research is always in progress.

Learn more biomedcentral.com/submissions 